\title{
Nonamplified 100Gbps Doubly Differential QPSK Optical Signal Transmission Over 80 km SSMF Without Carrier Recovery
}

\author{
Tingting Zhang, Christian Sanchez, Abdallah A. I. Ali, Andrew Ellis \\ Aston Institute of Photonic Technologies (AIPT) \\ Aston University \\ Birmingham, UK
}

\begin{abstract}
We numerically demonstrate a nonamplified 100-Gb/s doubly differential QPSK signal transmission over 80-km SSMF without carrier recovery or chromatic dispersion compensation. The receiver sensitivity after $80-\mathbf{k m}$ SSMF transmission was below $-28.8 \mathrm{dBm}$ for frequency offsets up to $2 \mathrm{GHz}$.
\end{abstract} Keywords-Coherent communications; Fiber optics
communications; Modulation

\section{INTRODUCTION}

The spread of bandwidth-hungry services such as broadband mobile communication and cloud computing has prompted the need of large data rate transmission in data center, access and metro networks. Intensity modulation direct detection (IM-DD) has long been regarded as the most costeffective solution for short-reach transmission. However, for long-haul $(\geqslant 80 \mathrm{~km})$ high-capacity $(\geqslant 40 \mathrm{~Gb} / \mathrm{s})$ transmission, optical amplification is required in IM-DD systems to ensure sufficient receiver sensitivity [1]. By contrast, digital coherent receiver using a high-power local oscillator (LO) and powerful digital signal processing (DSP) outperforms direct detection in terms of the receiver sensitivity, system reach and transmission capacity [2]. Nevertheless, in addition to a more complex optical frontend, the use of DSP functions to compensate for frequency offset (FO), phase noise and transmission impairments results in increased system complexity and power consumption. Therefore, an alternative compromise solution with reduced cost and power consumption, which does not significantly degrade the transmission performance, could be a promising candidate for next generation networks.

In this paper, we numerically demonstrate a $100-\mathrm{Gb} / \mathrm{s}$ polarization-division-multiplexed (PDM) doubly differential quadrature phase shift keying (DDQPSK) system using heterodyne detection and simplified DSP for $80-\mathrm{km}$ standard single mode fiber (SSMF) transmission without optical amplification. Doubly differential encoding [3] enables FO estimation and phase noise compensation to be eliminated from the DSP stack. Digital dispersion compensation is also avoided by adopting subcarrier multiplexing (SCM) [4] with tandem single sideband (TSSB) modulation to provide adequate dispersion tolerance. Numerical results show that line rate of $108 \mathrm{~Gb} / \mathrm{s}$, net bit rate of $100 \mathrm{~Gb} / \mathrm{s}$ with $7 \%$ hard-decision forward error correction (HD-FEC) overhead, has been achieved. The receiver sensitivity at FEC threshold of $3.8 \times 10^{-3}$ after $80-\mathrm{km}$ SSMF transmission was below $-28.8 \mathrm{dBm}$ for FOs up to $2 \mathrm{GHz}$, indicating 21.9-dB power budget for the launch power of $-6.9 \mathrm{dBm}$. Despite no carrier recovery, the sensitivity penalty caused by non-zero FO (up to $2 \mathrm{GHz}$ ) was negligible for different system reaches (0-km, 40-km and 80-km SSMF).

\section{SimUlation SetUP}

The simulation setup for the proposed PDM-DDQPSK system is shown in Fig. 1. For each polarization, four independent $2^{15}-1$ pseudo random binary sequences (PRBSs) were firstly bit wise Gray-coded and then doubly differentially (DD) precoded before being up-sampled to 8 Samples/symbol. Square root raised cosine (SRRC) filters with 0.5 roll-off factor and 3-dB bandwidth of $15.8 \mathrm{GHz}$ were applied for pulse shaping. The generated DDQPSK signals with the same symbol length of 65536 were separately modulated onto a 12 $\mathrm{GHz}$ RF subcarrier utilizing ideal IQ mixers. To generate TSSB signals in the optical domain, the up-converted DDQPSK signals were firstly input to orthogonal input ports (port 1 and 2) of four-port 90 degree hybrids (known as branchline combiners). Then the combined output signals (port 3 ) and their Hilbert transforms (port 4) were uploaded to VPI TransmissionMaker V9.5 to drive the IQ modulators with 5-V half-wave voltage and 6-dB insertion loss. Both submodulators were biased at null point with $90^{\circ}$ optical phase shift in between. Note that due to different transfer characteristics of the four-port hybrid, the two signals (shown in red in Fig. 1) before the IQ mixer connected to port 2 of the 90 degree hybrid were exchanged in order to maintain the same overall logical transfer function.

A $100-\mathrm{kHz}$ continuous wave $(\mathrm{CW})$ laser $(1550 \mathrm{~nm}, 9 \mathrm{dBm})$ modeled in VPI was utilized in the transmitter, whilst the other $100-\mathrm{kHz}$ laser $(18 \mathrm{dBm})$ was used as the LO in the receiver. Since heterodyne detection generated double sideband signal, the target frequency of the LO were set to be $24 \mathrm{GHz}$ lower than that of the transmitter to avoid signal overlapping. Typical tunable laser sources may be readily tuned to within $2 \mathrm{GHz}$ of the target wavelength, which was the maximum FO considered in our simulation. Two $43-\mathrm{GHz}$ balanced photo-detectors (BPDs) with responsivity of $0.45 \mathrm{~A} / \mathrm{W}$, shot noise and thermal noise current of $40 \mathrm{pA} / \sqrt{\mathrm{Hz}}$ were used for optoelectronic conversion. Two RF signals $(12 \mathrm{GHz}$ and $36 \mathrm{GHz}$ ) were respectively employed for down-conversion. The downconverted in-phase and quadrature signals of each sub-channel were combined together as a single DDQPSK signal before the SRRC low-pass filter, which had a 0.5 roll-off factor and 3-dB bandwidth of $20.42 \mathrm{GHz}$. After down-sampling to 2 Samples/symbol, 7-tap adaptive finite impulse response filters 


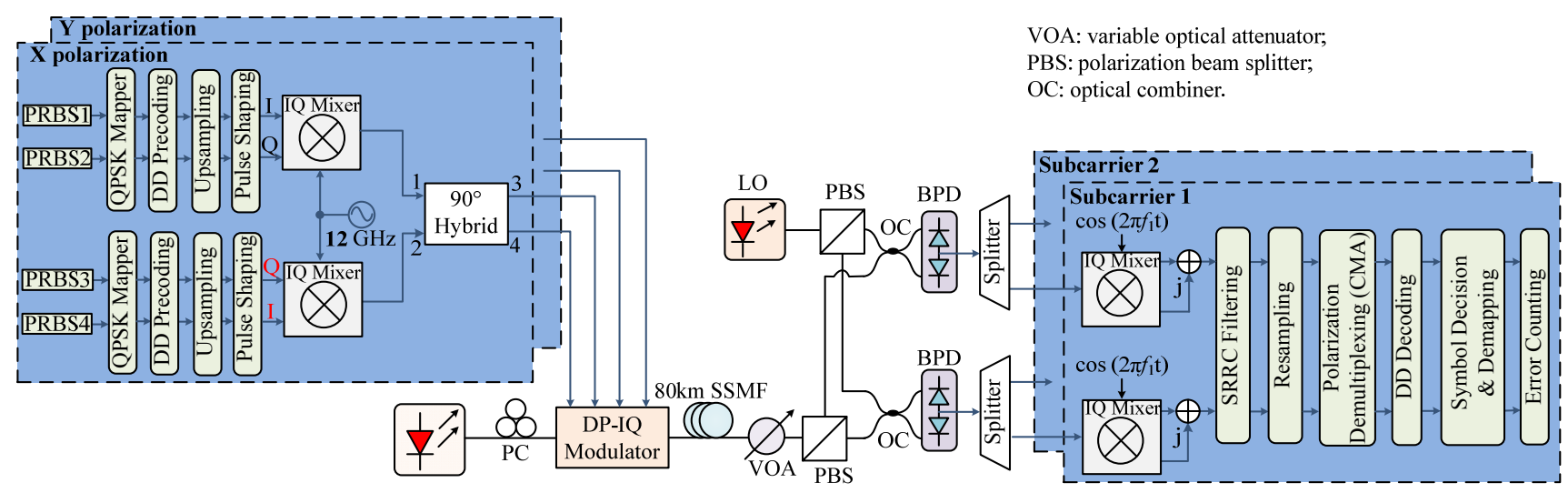

Figure 1. Simulation setup for 100-Gb/s PDM-DDQPSK system for 80-km SSMF transmission.

with tap weights optimized through the constant modulus algorithm (CMA) were utilized for polarization demultiplexing. The final error counting was implemented and summed over all subcarriers and polarizations after DD decoding, symbol decision and de-mapping. Note that no carrier recovery or dispersion compensation were used in the receiver, leading to simplified DSP functions.

\section{RESULTS AND DISCUSSION}

Compared with the antialiasing low-pass filter embedded in the resample Matlab function, the SRRC low-pass filter used in the receiver had smaller bandwidth, restricting the tolerable FO, which was still larger than the target FO (2 $\mathrm{GHz}$ ). The simulation was run 8 times, each with different polarization evolutions, and the error bars in Fig. 2 were obtained by calculating the mean and standard deviations of the bit-error-rates (BERs) from the 8-runs. Fig. 2 shows that even without carrier recovery and chromatic dispersion compensation, the sensitivity (at FEC threshold of $3.8 \times 10^{-3}$ ) after $80-\mathrm{km}$ SSMF transmission was below $-28.8 \mathrm{dBm}$, giving a power margin of $5.9 \mathrm{~dB}$ for $-6.9-\mathrm{dBm}$ launch power. Compared with back-to-back (BTB) transmission, the receiver sensitivity after $80-\mathrm{km}$ SSMF transmission was degraded by $0.5 \mathrm{~dB}$, while the sensitivity for $40 \mathrm{~km}$ transmission was almost unaffected. The sensitivity penalty

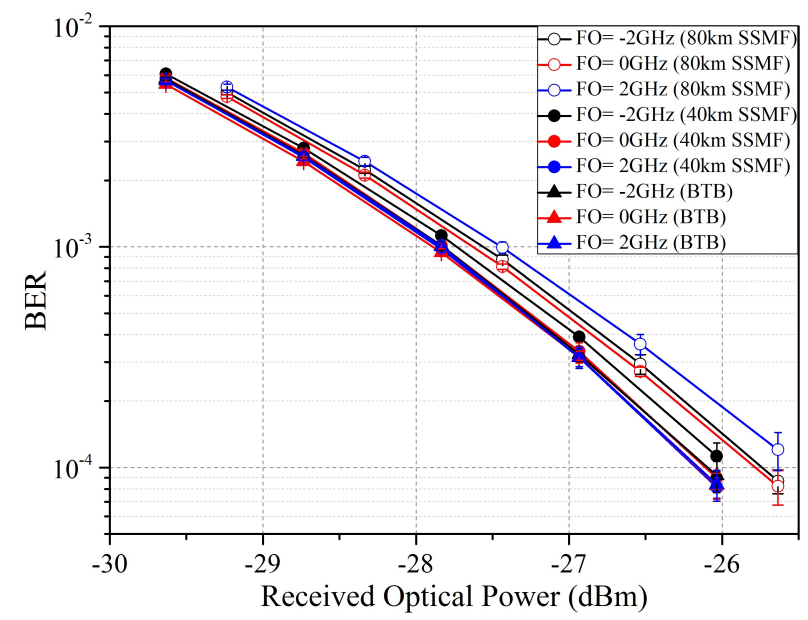

Figure 2. BER curves for the 100-Gb/s PDM-DDQPSK signal BTB, 40$\mathrm{km}$ and $80-\mathrm{km}$ SSMF transmission with FOs up to $2 \mathrm{GHz}$. caused by nonzero FO (up to $2 \mathrm{GHz}$ ) for different system reaches $(0-\mathrm{km}, 40-\mathrm{km}$ and $80-\mathrm{km}$ SSMF) was negligible (around $0.2 \mathrm{~dB}$ ), indicating the advantage of DD encoding.

\section{CONCLUSION}

We have numerically demonstrated a $100-\mathrm{Gb} / \mathrm{s}$ PDMDDQPSK system transmission over $80-\mathrm{km}$ SSMF without optical amplification, dispersion compensation or carrier recovery. Compared with full digital coherent receiver, the proposed heterodyne receiver has lower cost due to the elimination of the optical hybrid and two BPDs, and is more power-efficient because of the simplified DSP functions. The receiver sensitivity (at BER of $3.8 \times 10^{-3}$ ) after $80-\mathrm{km} \mathrm{SSMF}$ transmission was below $-28.8 \mathrm{dBm}$, giving a power margin of $5.9 \mathrm{~dB}$. Despite no carrier recovery, the non-zero FO (up to $2 \mathrm{GHz}$ ) induced sensitivity penalty for different system reaches $(0-\mathrm{km}, 40-\mathrm{km}$ and $80-\mathrm{km}$ SSMF $)$ is negligible.

\section{ACKNOWLEDGMENT}

This work was partially supported by the EPSRC projects PEACE (EP/L000091/1), UPON (EP/M005283/1), Silicon Photonics for Future Systems (56474), and by the EU project INVENTION (659950). The data underlying this publication can be found at http://doi.org/10.17036/researchdata.aston.ac.uk.00000245.

\section{REFERENCES}

[1] W. Yan, L. Li, B. Liu, H. Chen, Z. Tao, T. Tanaka, T. Takahara, J. Rasmussen, and D. Tomislav, "80 km IM-DD transmission for 100 $\mathrm{Gb} / \mathrm{s}$ per lane enabled by DMT and nonlinearity management," in Optical Fiber Communication Conference 2014, paper M2I.4.

[2] O. Bertran-Pardo, D. Mongardien, P. Bousselet, P. Tran, H. Mardoyan, I. Brylski, J. Renaudier, and H. Bissessur, "Transmission of $2.6 \mathrm{~Tb} / \mathrm{s}$ using $100-\mathrm{Gb} / \mathrm{s}$ PDM-QPSK paired with a coherent receiver over a 401-km unrepeatered link," IEEE Photon. Technol. Lett, vol. 21, pp. 1767-1769, 2009.

[3] A. J. Walsh, H. Shams, J. Mountjoy, A. Fagan, J. Zhao, L. P. Barry and A. D. Ellis, "Demonstrating doubly-differential quadrature phase shift keying in the optical domain," IEEE Photon. Technol. Lett., vol. 25, pp. 1054-1057, 2013.

[4] F. A. Gutiérrez, P. Perry, E. P. Martin, A. D. Ellis, F. Smyth, and L. P. Barry, "All-analogue real-time broadband filter bank multicarrier optical communications system," J. Lightw. Technol., vol. 33, pp. 5073-5083, 2015. 